Case Report

\title{
Prone Anesthesia for a Super-Super Morbidly Obese Patient Undergoing Neurosurgery: A Case Report
}

Ian W. Stone ${ }^{1}$, Ashim Gupta ${ }^{2,3,4,5}$, Hugo C. Rodriguez ${ }^{1,2}$, R. Justin Mistovich ${ }^{6}$, Manu Gupta ${ }^{3}$, Anish G. Potty ${ }^{2,7}$, Jorge R. Serrato ${ }^{8, *}$

1. University of the Incarnate Word School of Osteopathic Medicine, San Antonio, Texas, USA; EMails: istone@student.uiwtx.edu; hcrodrig@student.uiwtx.edu

2. South Texas Orthopaedic Research Institute, Laredo, Texas, USA; ashim6786@gmail.com; anishpotty@gmail.com

3. Future Biologics, Lawrenceville, Georgia, USA; E-Mail: manu6771@yahoo.co.in

4. Biolntegrate, Lawrenceville, Georgia, USA

5. Veterans in Pain, Los Angeles, California, USA

6. Department of Orthopaedics, School of Medicine, Case Western Reserve University, Cleveland, Ohio, USA; E-Mail: justin@mistovich.net

7. Laredo Sports Medicine Clinic, Laredo, Texas, USA

8. Laredo Medical Center, Laredo, Texas, USA; E-Mail: jorge.serrat@gmail.com

* Correspondence: Jorge R. Serrato; E-Mail: jorge.serrat@gmail.com

OBM Neurobiology

2020, volume 4, issue 2

doi:10.21926/obm.neurobiol.2002064
Received: May 03, 2020

Accepted: June 23, 2020

Published: June 24, 2020

\section{Abstract}

Obesity is on the rise around the world, carrying along with it a wide range of comorbidities and complications. We report on a super-super morbidly obese 43-year-old female, with a body mass index $(\mathrm{BMI})$ of $77.85 \mathrm{~kg} / \mathrm{m}^{2}(173 \mathrm{~cm}, 233 \mathrm{~kg})$, who presented for a scheduled suboccipital craniectomy with C-1 laminectomy secondary to symptoms from an ArnoldChiari type-I malformation. Already a challenging type of surgery, due to the patient's extreme BMI and positioning, the surgery became more difficult. There are many potential complications for general anesthesia in the obese patient, including a difficult airway, postoperative breathing problems due to obstructive sleep apnea (OSA), risk for cardiovascular events, and complications due to changes in drug pharmacokinetic/dynamic properties. In addition to the complications associated with anesthesia, the patient needed to be prone

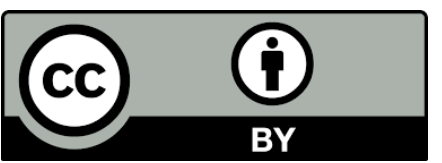

(C) 2020 by the author. This is an open access article distributed under the conditions of the Creative Commons by Attribution License, which permits unrestricted use, distribution, and reproduction in any medium or format, provided the original work is correctly cited. 
and positioned using Mayfield pins. Despite these challenges, there were neither anesthesia nor surgical complications. Thus, while careful considerations and informed consent should be obtained, BMI alone should not deter one from performing a procedure.

\section{Keywords}

Morbid obesity; obese patient; anesthesia; peri-operative complications; prone position

\section{Introduction}

The incidence of obesity is rising worldwide and has nearly tripled since 1975 [1]. Increased BMI is associated with over 230 comorbidities, is an increase in all-cause mortality, and is associated with development of cardiovascular disease [2]. Specifically, individuals with obesity and increased central fat are at increased risk for type-2 diabetes mellitus, metabolic syndrome, hypertension, heart disease (including coronary artery disease, heart failure, and myocardial steatosis), venous thrombosis, sleep apnea, and many other conditions [2]. Interestingly enough, there is a so-called 'obesity paradox' wherein obesity is somehow protective against mortality in certain conditions, although this has been under some contention [3]. A BMI between 18.5-24.9 is considered normal, 25-29.9 is overweight, $30-39.9$ is obese, $40-49.9$ is morbidly obese, $50-59.9$ is super morbidly obese, and 60+ is super-super morbidly obese. Despite these complications and the known changes in pulmonary and cardiac physiology, prone positioning has been shown to increase pulmonary function for obese patients during general anesthesia [4]. Difficulty with mask ventilation must also be considered in this patient population, as $B M I>26 \mathrm{~kg} / \mathrm{m}^{2}$ is one of the five criteria established by Han et al [5] for grading difficult mask ventilation.

While preoperative optimization focused on addressing associated comorbidities helps improve the success of anesthesia, such actions were not indicated in this case. Some of the most common comorbidities to consider would be OSA, which can lead to significant pulmonary hypertension, and various associated cardiovascular problems. Our patient's medical history was significant only for hypothyroidism and hypertension. One preparatory, additional step we undertook was to bring the patient to the operating room the day prior to her scheduled surgery. While awake, we determined optimal positioning on the operating table. The patient was placed in a modified swimming position with padding under her thighs and chest to prevent the table from transmitting that pressure into her cardiovascular and respiratory systems. We adjusted the padding to find maximal relief of pressure on the patient's abdomen, assessed for other potential points of increased pressure, and evaluated the patient's ability to breathe deeply.

\section{Case Report}

Published with the written consent of the patient.

A 43-year-old female presented with severe symptoms from an Arnold-Chiari Malformation type-I. Symptoms included severe headaches, tingling to the right upper and lower extremities, and associated right-sided weakness. Medical history included hypertension and hypothyroidism. Surgical history included gastric bypass surgery with a history of social smoking and drinking. 
Patient had no drug allergies, a normal electrocardiogram, complete blood count, basic metabolic panel, and chest X-ray pre-operatively.

The patient's airway exam was assessed. She displayed a Mallampati score of 1 with full visualization of the tonsillar pillars and intact dentition. Patient's thyromental distance was greater than $4 \mathrm{~cm}$. She was able to fully open her mouth with no deficits in temporomandibular joint mobility. Neck motion was limited due to pain. The patient had been intubated previously with no history of difficult intubation. The day before surgery, the patient's position on the operating table was assessed as well as any possible pressure points and her depth of breathing. There was adequate lung excursion and the patient's neck was assessed in the prone position for surgical exposure.

On the day of surgery, compression stockings and sequential compression devices were placed for deep venous thrombosis prophylaxis. American Society of Anesthesiologists standard monitors were placed. Patient was given oxygen for 5 minutes for complete denitrogenation. Patient was placed with a small degree of reverse Trendelenburg position for better ventilation. Due to a previously described favorable airway, we decided on intubation after induction of general anesthesia. We then administered midazolam $2 \mathrm{mg}$ for anxiolysis followed by $150 \mathrm{mg}$ of lidocaine, $100 \mathrm{mcg}$ of fentanyl, and $200 \mathrm{mg}$ of propofol for induction. Patient was masked using an oral airway without any difficulty. Paralysis was achieved with $150 \mathrm{mg}$ succinylcholine. Patient's trachea was then intubated with video laryngoscopy via an $8.0 \mathrm{~mm}$ tracheal tube at $23 \mathrm{~cm}$ at the lips with no difficulty while maintaining c-spine stability. $50 \mathrm{mg}$ of rocuronium was administered for long term paralysis. A left radial arterial line was placed using ultrasound due to potential for hemodynamic changes intraoperatively.

Nine members of the surgical team assisted in turning the patient into a bariatric bed. Her eyes, ears, and nose were positioned on a prone positioning pillow free of pressure. Neck was neutral and the pressure points were padded (Figure 1). Two chest rolls were placed horizontally with the patient's chest situated in-between. A roll of blankets was placed on the patient's hip/leg area allowing the diaphragm to be pressure free. The patient's tidal volumes were assessed before proceeding with further positioning. The ventilator's pressure control was titrated to $27 \mathrm{~cm} \mathrm{H}_{2} \mathrm{O}$ with tidal volume at $650 \mathrm{~mL}$ and a respiratory rate of $12 \mathrm{~b} / \mathrm{min}$. Patient was in total intravenous anesthesia (TIVA) throughout the surgical procedure. Propofol was set at $120 \mathrm{mcg} / \mathrm{kg} / \mathrm{min}$ and remifentanil set to $0.12 \mathrm{mcg} / \mathrm{kg} / \mathrm{min}$. One hundred kilograms was used as the body weight subjectively.

The patient was stable, ventilated, and appropriately positioned. She was moved cephalad and placed on Mayfield pins for her surgical procedure. Suboccipital decompression with C-1 laminectomy was performed. Patient was prepared for extubation with 4/4 on TOF (train-of-four) and sugammadex $400 \mathrm{mg}$ was given for reversal. The patient was extubated and transferred to the post-anesthesia care unit (PACU) in stable condition. A non-opioid analgesia strategy was used for post-operative pain control. Infiltration of local anesthetic $0.5 \%$ bupivacaine with epinephrine was administered by the surgeon. In PACU, $1 \mathrm{~g}$ of Ofirmev was given. No complications were noted from surgery or anesthesia. The patient's neurological symptoms improved, and she was discharged home two days later. 


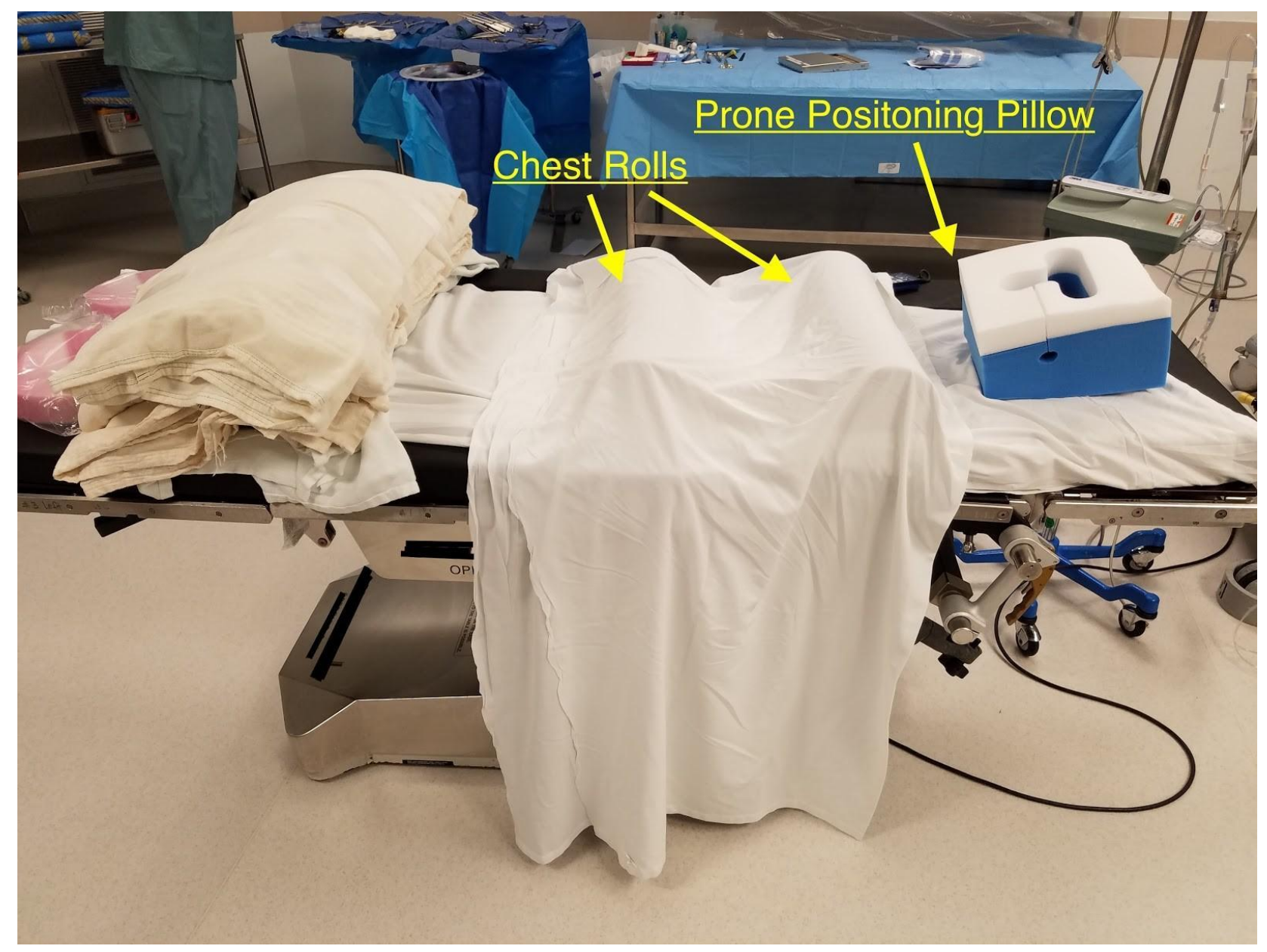

Figure 1 Prone Anesthesia Setup. Bariatric bed with 1000lb weight limit, with a prone positioning pillow placed at the head of the table. Two chest rolls placed horizontally across the table at the midline. A bundle of operating room blankets were situated near the caudal end.

\section{Discussion}

With the rates of obesity rising throughout the world, an increased ability to detect those who will prove difficult anesthesia cases is paramount. Some projections estimate that by 2030 up to $57.8 \%$ of the world's population could become either overweight or obese [6]. Even though our patient had a BMI much greater than that encountered in the literature, the operation proceeded with minimal problems: intubation with video laryngoscopy proved to be non-difficult, ventilation was adequate, intraoperative vital signs remained stable, extubation was without problems, and her PACU stay was uneventful. Considering there is increased morbidity and mortality for the morbidly obese, it is important to be vigilant and be able to anticipate anesthetic complications.

Prone anesthesia has been associated with certain complications resulting from increased pressure on anterior structures [7]. As such, proper positioning is critical and assessing for any areas of increased pressure must be addressed to prevent the development of sores or nerve entrapment. If proper positioning and padding is not used, intra-abdominal pressures rise, which can result in compression of the inferior vena cava, leading to venous stasis. Chest roll usage 
allows the abdomen to hang freely, decreasing visceral pressure and congestion on the cardiovascular and respiratory systems [7].

The prevalence of OSA in the general population is about $20 \%$ and severe OSA is undiagnosed in about $10-20 \%$ of obese patients [3]. There is great importance in screening for this condition in this patient population. One method of screening is using the Snoring, Tiredness, Observed apnea, high blood Pressure (STOP)-Body mass index, Age, Neck Circumference and Gender (BANG) screening tool. The preoperative evaluation should consist of a thorough review of medical records (sleep studies preferred), a patient/family interview, screening protocol, and a physical examination [8]. Both the surgeon and anesthesiologist need to plan well in advance of the surgery to allow for an appropriate perioperative course of action. Preoperative preparation is aimed at optimizing the patient's physical status and includes preoperative continuous positive airway pressure or noninvasive positive pressure ventilation, preoperative use of mandibular advancement or oral appliances, and preoperative weight loss, although there is insufficient literature on these methods' efficacy [8]. In order to limit systemic opioid usage in this patient population, regional anesthetic techniques are preferred. Postoperative management should focus on postoperative analgesia, oxygenation, patient positioning (in a non-supine position), and monitoring [8]. TIVA was the maintenance of choice to prevent increased intra-cranial pressure with inhalational agents. Also, due to the extreme BMI, TIVA was used because of the potential for inhalational agents to dissolve in adipose tissue and cause prolonged emergence and even cause the patient to become re-anesthetized due to hypoventilation.

Obese patients, in order to maintain cardiovascular demands, have increased cardiac output and blood pressure, leading to a significant amount of cardiac workload [9]. The increased amount of adipose tissue in this patient population increases the body's metabolic demands [10]. These increased demands on the physiology of the patient are important to consider while preparing for anesthetic complications. Invasive cardiac testing as well as cardiopulmonary exercise testing may be needed $[9,10]$. Exercise or pharmacological stress tests, stress echocardiography, and myocardial nuclear imaging are all examples of the cardiac testing that can be utilized. These allow the clinician to assess the patient for cardiac complications and optimization of their condition. Obesity increases the risk for metabolic comorbidities which can ultimately lead to coronary artery disease. Increased cardiovascular demands due to obesity and coronary artery disease can lead to ischemic cardiac events. The fatty infiltration of the conducting system in the heart leads to increased risk of arrhythmias such as atrial fibrillation [9]. With that in mind, sudden cardiac death must be considered both in preoperative planning and informed consent discussions [9]. The degree of Q-T prolongation has also been shown to be increased in this population and should be taken into account with adverse drug reactions related to anti-microbial and anti-emetic medications [9].

There are also important pharmacokinetic and pharmacodynamic changes to consider in the obese patient population. Drugs are usually dosed based on the patient's weight/size and the physical condition that they are in. However, in obese patients, adipose tissue and lean body mass (LBM) do not increase proportionally, with adipose tissue increasing proportionally to total body weight (TBW) and the ratio of LBM to TBW decreasing as TBW increases [11]. This leads to changes in volume of distribution, binding, and elimination of drugs, requiring the need to individualize the course of anesthesia. Obesity also affects drug pharmacokinetics by inducing changes in hemodynamic status, regional blood flow, and plasma protein binding. Some drugs, 
such as thiopental, should be dosed according to LBM instead of TBW as required doses may be much less than in normal-weight patients [11]. Propofol has been shown to be both safe and effective for TIVA in the morbidly obese, however this drug should be dosed according to actual body weight. Remifentanil is likewise a suitable agent as it is rapidly cleared by blood and tissue esterases, resulting in quick onset coupled with rapid clearance, and should be dosed according to LBM [11].

\section{Conclusion}

The morbidly obese patient can pose difficulties for anesthesiologists, but the BMI in and of itself should not deter one from performing a needed procedure and should be evaluated on a case-by-case basis. Certain considerations must be observed when dealing with the obese patient population, especially when they have particular comorbidities. These comorbidities need to be assessed and addressed by all members of the healthcare team in the pre-, intra-, and postoperative phases. Some of these can be optimized in the preoperative period in order to ensure success of both the anesthesia and the surgery. In addition, the positioning of the patient may need to be altered to allow for proper physiological mechanics intraoperatively.

\section{Author Contributions}

IWS, AG, and JRS conceptualized and designed the study. JRS performed the anesthesia. IWS, AG, HCR, and JRS wrote the manuscript draft. IWS, AG, HCR, RJM, MG, AGP, and JRS edited the manuscript. All authors read and approved the final manuscript.

\section{Competing Interests}

The authors have declared that no competing interests exist.

\section{References}

1. World Health Organization. Obesity and overweight, 2018. [2020 February 20]. Available from: https://www.who.int/en/news-room/fact-sheets/detail/obesity-and-overweight

2. Perreault L. Overweight and obesity in adults: Health consequences, 2019. [2020 February 20]. Available from: https://www.uptodate.com/contents/overweight-and-obesity-in-adultshealth-consequences

3. Lang LH, Parekh K, Tsui BYK, Maze M. Perioperative management of the obese surgical patient. Br Med Bull. 2010; 124: 135-155. https://doi.org/10.1093/bmb/ldx041

4. Pelosi $P$, Croci $M$, Calappi E, Mulazzi D, Cerisara $M$, Vercesi $P$, et al. Prone positioning improves pulmonary function in obese patients during general anesthesia. Anesth Analg. 1996; 83: 57883. https://doi.org/10.1097/00000539-199609000-00025

5. Han R, Tremper K, Kheterpal S, O'Reilly M. Grading scale for mask ventilation. Anesthesiology. 2004; 101: 267.

6. Kelly T, Yang W, Chen CS, Reynolds K, He J. Global burden of obesity in 2005 and projections to 2030. Intern J Obes. 2008; 32: 1431-1437. https://doi.org/10.1038/ijo.2008.102 
7. Kwee MM, Ho Y, Rozen WM. The prone position during surgery and its complications: $A$ systematic review and evidence-based guidelines. Intern Surg. 2015; 100: 292-303. https://dx.doi.org/10.9738\%2FINTSURG-D-13-00256.1

8. Lang LH, Parekh K, Tsui BYK, Maze M. Practice guidelines for the perioperative management of patients with obstructive sleep apnea: An updated report by the American Society of Anesthesiologists Task Force on Perioperative Management of patients with obstructive sleep apnea. Anesthesiology. 2014; 120: 268-286. doi: https://doi.org/10.1097/ALN.0000000000000053

9. Nightingale CE, Margarson MP, Shearer E, Redman JW, Lucas DN, Cousins JM, et al. Perioperative management of the obese surgical patient 2015. Anaesthesia. 2015; 70: 859-876. https://dx.doi.org/10.1111\%2Fanae.13101

10. Pedoto A. Lung physiology and obesity: Anesthetic implications for thoracic procedures. Anesthesiol Res Pract. 2012; 2012: 154208. https://dx.doi.org/10.1155\%2F2012\%2F154208

11. Leykin Y, Miotto L, Pellis T. Pharmacokinetic considerations in the obese. Best Pract Res Clin Anaesthesiol. 2011; 25: 27-36. https://doi.org/10.1016/j.bpa.2010.12.002

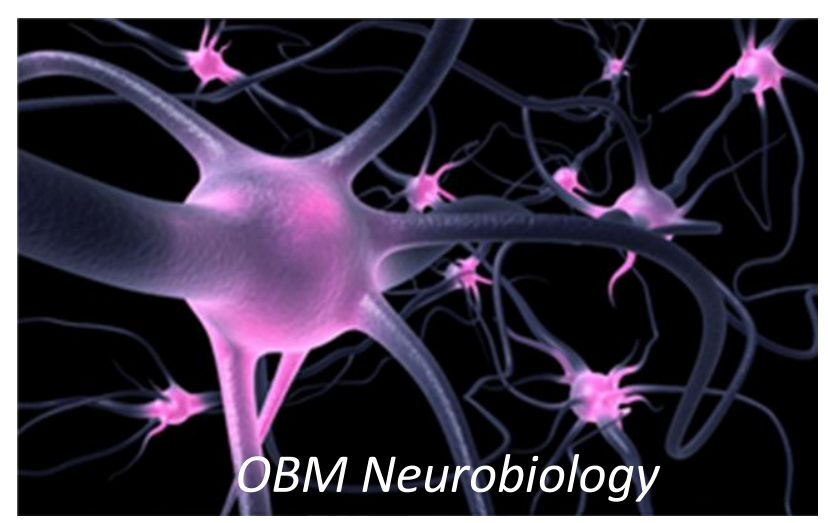

Enjoy OBM Neurobiology by:

1. Submitting a manuscript

2. Joining volunteer reviewer bank

3. Joining Editorial Board

4. Guest editing a special issue

For more details, please visit:

http://www.lidsen.com/journals/neurobiology 